\title{
Research results of spatial mechanisms and directions of their application in farming machinery
}

\author{
Alexander P. Mudrov ${ }^{1}$, Alexander G. Mudrov², Sergey M. Yakhin ${ }^{1}$, and Gennady V. Pikmullin 1,* \\ ${ }^{1}$ Kazan State Agrarian University, 420015 Kazan, Russia \\ ${ }^{2}$ Kazan State University of Architecture and Engineering, 420043 Kazan, Russia
}

\begin{abstract}
The paper touches upon the issue of spatial hinge mechanisms with a special structure, developed on the basis of Bennett's spatial four-bar linkage. The interest in the study of such mechanisms and their use in technology is constantly growing, since they are distinguished by a high efficiency, the transmission accuracy of motion law, the rigidity of the structure and the ability to transfer high loads. In addition, the hinges of the mechanisms are represented only by rotating pairs, which in the design of the hinge units allows the use of rolling bearings and reliably isolate the units from the external environment. The authors give several areas of application of mechanisms as basic and independent devices in technology, including the agro-industrial complex.
\end{abstract}

\section{Introduction}

Spatial mechanisms are widely used in various branches of technology: appliances, textile technology, airplanes, tractors, automobiles and so on, but they are especially used in agricultural machines. The academician V.P. Goryachkin wrote: "Spatial mechanisms are not developed enough so far, since they are relatively rarely used, but their use in agricultural machines and implements is very wide (mowers, reapers, sheaves, rakes, tedders, plows)" [1].

It is necessary to note that V.P. Goryachkin was the first in Russia to study the theory of spatial mechanisms, who performed in 1914 a kinematic and force analysis of a reaping machine rake.

In his fundamental work «Agricultural Mechanics », he devoted a separate paragraph to these mechanisms. Currently, the the list of machines using spatial mechanisms, specified by V.P. Goryachkin, includes grain, potato and sugar beet harvesters, balers, planters and others. The schemes using four-, five- and six-bar mechanisms are gave been implemented.

Four-bar linkage mechanisms are used both with a crank and balancer and with two balancers. The example of the use of a four-bar linkage system with a crank and a balancer is the drive of the sickle knife of the cutter bar of a combine harvester, and example of the use of two balancers is the safety mechanism hook of piston stop of a baler; the deck mechanism of the combine harvester and the copier-driver mechanism of the beet harvester $[2,3,4]$. Spatial five-bar linkage mechanisms, the kinematics of which was studied in detail by G.D. Ananov [5], are used to convert the rotary motion of the crank into the back-and-forth motion of the balance bar or into the back-and-forth motion of the slider, for example, in the drive of the mower knife.

Six-bar mechanisms are used in tedders, or active plow shares, which, in addition to plowing the soil layer, has an additional dynamic effect on it. In addition, Cardan-Hooke hinges can be used in agricultural machines, spherical mechanisms with a swinging washer and a fork [6].

Most of the spatial mechanisms have spherical, spherical with a finger or ball-cylinder kinematic pairs in their structure, which complicates the design, reduces its processability and the service life of a device based on such mechanisms. It is possible to simplify the design, increase the reliability, performance and service life of a device using spatial mechanisms with only rotary kinematic pairs, created on the basis of the Bennett mechanism.

The purpose of the research is to develop a theory of design, creation and research of new reliable, efficient devices for agricultural purposes based on spatial hinge mechanisms with some rotational pairs.

\section{Materials and Methods}

The use of some rotational pairs in the spatial mechanism allows constructive design of hinge units on rolling bearings, reliably isolating them from the effects of unfavorable working environment. This aspect not only increases the reliability and durability of the device, but also increases its efficiency and transmitted power load.

However, it is impossible to obtain a spatial mechanism simply attaching bar without taking into account their geometric parameters and their consistency. Mechanical scientists, realizing the great 
advantage of a mechanism with only rotational pairs, tried to create such mechanisms, but none of them managed to obtain a workable sample, the mechanisms did not rotate [7].

Only in 1903, English mathematician Jeffrey Thomas Bennett (1868-1943) theoretically described a four-bar spatial mechanism (later named after him), the hinge axes of which intersected at certain angles. Bennett found a relationship between the angles (tangents of half angles) of rotation of the bars associated with the mechanism rack and its graphic interpretation, but he could not make a model of the mechanism (the mechanism did not rotate) and for this reason he doubted its practical use.

Later, many national and international scientists investigated this mechanism, theoretically formed two or three more mechanisms, but they could not produce either a model or a full-scale sample of at least one of the mechanisms of this group. Only 70 years after the theoretical description of Bennett's mechanism, the scientists of the Department of Machine Elements of Kazan Agricultural Institute, professors B.V. Shitikov and P.G. Mudrov solved the problem of model creation and a full-scale sample [8].

They found out what was the problem of education, research, production and implementation of the mechanisms of this group. Firstly, the mechanisms of this group can not be created with the usual combination of bar. Secondly, these mechanisms are statically indefinable (thrice, twice, and once) and formally should not exist, therefore scientists and practitioners do not pay due attention to them. Thirdly, the mechanisms can exist with consistent kinematic and linear parameters and the relationships between them that need to be determined. Fourthly, the production of mechanisms and devices based on them has a special specificity, without which the creation of a workable structure is impossible. Fifthly, neither in our country nor abroad there are scientific schools that would closely deal with these mechanisms.

\section{Results}

It is necessary to say that spatial mechanisms with rotational pairs received their start in life due to the scientists of the Department of Theory of Mechanisms and Machines (now the Department of General Engineering Disciplines) of Kazan State Agrarian University, whose developments are priority at national and international levels. Despite the rapid development of technology, this group of mechanisms has been defined and established practically over the past 30 years by the staff of only one department. During this time, the theory of spatial mechanisms with rotational kinematic pairs was developed [8-11].

The problem of the creation multi-bar mechanisms with given properties was solved, four methods of forming five-, six-, seven-, multi-bar and differential mechanisms were proposed. The methods for the kinematic and dynamic research of mechanisms of all groups were developed, the external pressure of the moving bars on the bed was studied, several methods for balancing the mechanisms were proposed. The synthesis of mechanisms has been developed depending on the functional purpose. The technology of producing devices for small-scale and individual production was obtained. The theory of creation of spatial mixing devices and 7 groups of objects of new technology was developed [9, $10]$.

More than one hundred new mechanisms and devices based on them was proposed and developed, protected by 123 copyright certificates and patents for inventions. Bennett's mechanism laid the foundation for a new scientific direction in the theory of spatial mechanisms and became a building module in the formation of new types of such mechanisms and devices based on them.

On the basis of Bennett's mechanism alone, thirty-four devices at the level of inventions were created, and in total, according to the results of research, more than fifty devices were created and implemented, which were used in agricultural and industrial enterprises in Russia and Ukraine. The difficulty of the formation and development of mechanisms was compensated by a variety of useful properties, the number of which they exceeded other known mechanisms.

A theory for designing new apparatus with a stirrer, which carry out four types of mixing and processing of materials developed: in-cycle, out-of-cycle, combined and pulse. The use of this theory resulted in four directions of design and improvement of devices with a stirrer. The first is to impart intra-cycle variable rotation to the working blades; the second is the creation of a complex spatial movement for the blades inside the mixing chamber; the third is the use of several movable blades for mixing, moving according to different laws; the fourth is the design of mixers with two or more vertical working shafts moving according to different laws.

The theory of creating new spatial mixers was developed on the basis of the theory of designing apparatus with a stirrer including the proposed mixing methods. In the course of implementing the theory, seven new classification groups of spatial mixers were created: spatial inertial, spatial with a vertically located mixing tank (drum), with cantilever drum fastening, console multi-drum, spatial inertial double-drum, combined mixers and mixers with continuous operation [10].

In the course of a dynamic study of the created mixer designs, it was revealed that the maximum values of variable power loads acting on the links and hinge nodes of the mixer in one rotation of the driving crank reach $\varphi$ at certain values of the angle of its rotation. Thus, for example, in inertial mixers - at an angle $\varphi=0$ and $180^{\circ}$ and in cantilever mixers - at $\varphi=300-360^{\circ}$.

These values are used for practical strength calculations. Mathematical dependences were obtained to determine the balancing (driving) moment applied to the driving crank of the mixers, which are used in the calculation of their drives.

The equations are derived to determine the pressures of the mixer moving bars on its bed, the analysis of 
which allows developing six ways of balancing the mixers.

The first method consists in the redistribution of the mass of the drum to crank heads and use of a flywheel that has the same kinematic momentum with the driven crank, but rotating in the opposite direction.

The second is the use of additional drums in cantilever mixers.

The third consists in the setting of the second drum, the driven crank of which rotates in the opposite direction to the direction of rotation of the driven crank of the first drum.

The fourth is connected with the placement of the drive source and the converting mechanism on opposite sides of the drum in combined mixers.

The fifth method consists in the connection of two mirror structures of the mixers with a common drive shaft.

The sixth consists in the elimination of the driven crank and shifting the drum towards the hinge of the driving crank and installing the flywheel on its shaft.

A mathematical model for the movement of particles in a mixing tank performing complex spatial motion was developed. During the analysis of the model on a computer, the dependences of the rotational speed of the driving crank, mixing drum and mixer parameters for guaranteed volumetric movement of the mixed components were obtained. The nomograms to determine the optimal parameters of the mixer during its design were created.

The methods for testing the general theory of the creation of spatial mixing devices, mathematical models of mixers, as well as the mixing process in them were developed and experimentally confirmed in.

The integrated method for the solutions was embodied in the general theory of the creation of spatial mixing devices and devices for other areas of technology: transfer-converting mechanisms for agricultural machines, inertial motors, devices for driving piles, earth-moving machines, flies, rowing devices, machines for liquid washing and cleaning products.

In this paper, we briefly describe the main types of devices, grouped by types of technology based on spatial mechanisms.

Drives of agricultural machines: potato digger elevator drive (a.s. 695609), disk drive leveler of beet harvester (a.c. 808035), cutting device drive (a.c. 869649, 967353), hinge-link transmission mechanisms (AS 815365, 939817, 947536).

Spatial mixing devices: devices with a stirrer and mixer-tumbling devices ( 9 inventions of devices with a stirrer and 36 of mixers).

Devices for primary processing: devices for polishing and tumbling (a.c. 795958, 643305, 730548, 742114, 751593, 753611, 812535, 818831), devices for grinding sugar beet seeds (patents 2078489, 2080038), devices for dressing seeds (a.c. 950216, 1658859), devices for quenching products (a.c. 1169998, 1330182), devices for electroplating of parts (AS 1186706).
Washing and cleaning devices: devices for washing vehicles (a.c. 560778, 698809, 766675, 1011446), devices for liquid processing of products (a.c. 848509, 883210), devices for cleaning hollow products (a.s .1310044), devices for cleaning watch mechanisms (A.C. 1158969).

Grinding materials devices: hammer mill (a.c. 1502089, 1590144, 1650397), device for processing polymeric materials (a.c. 1211060), grinder (a.c. 7889871).

Inertial devices: device for driving piles (a.c. 1245656), inertial motor (a.c. 939817).

Devices for copying movements: earth-moving machine (a.c. 883248), device fors combing fur (a.c. 1472513), ornithopter wing drive (a.c. 1259665), rowing device for watercraft (a.c. 1235792), gripping device (a.c. 810976, 1333575).

In practical use, the mechanisms and devices based on them provide energy savings in all cases, due to: the first is high efficiency, for example, in the Bennett mechanism it is 0.97-0.98, in the five-bar devices is 0.96-0.97 and in six-bar devices is 0.95-0.96; the second is the complex spatial movement of the working bodies (containers, blades, etc.) with an uneven speed and at the same time an additional inertial effect, adjustable over a wide range. This helps to reduce the time of the process (mixing, pickling) more than 2 times, improve the quality of the final product (homogeneity of the mixture 98-99\%), reduce energy consumption for stirring by 3-6 times in comparison with existing devices of equal productivity.

The use of the Bennett mechanism, five-bar and sixbar mechanisms is carried out in the following characteristic directions:

1. Motion transmission between shafts, arbitrarily located in space. Now it requires two pairs of bevel wheels, or one pair of bevel wheels and a chain or belt drive. The drive by means of a spatial mechanism greatly simplifies the design of the transmission and the device as a whole, increases efficiency and reliability, and reduces energy consumption.

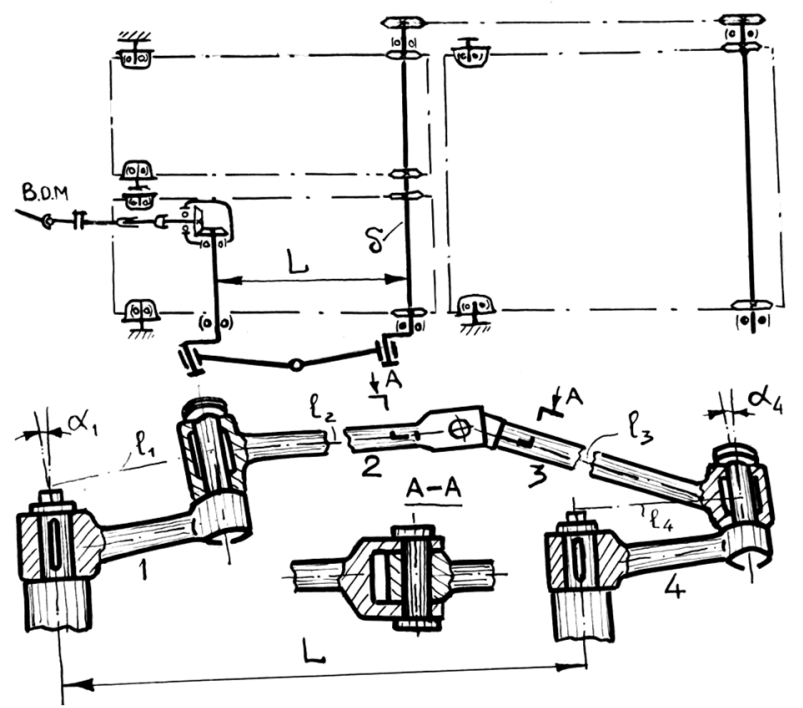

Fig. 1. Scheme of elevator drives of potato harvester 
For example, the use of a spatial mechanism in the drive of elevators of a potato harvester (Figure 1); the drive of the beet harvester leveler reduces energy consumption by 1.3-1.7 times in comparison with existing drives.

2. Transmission to the working bodies of various machines of intracycle variable kinematic velocity with a gear ratio equal to 1,2 and 3 . This property of mechanisms can be used to intensify various processes: mixing materials, separating soil, dimensionless processing of parts, washing and cleaning, etc.

As an example, figure 2 shows the drive of the washing platform on the basis of a spatial four-bar mechanism.

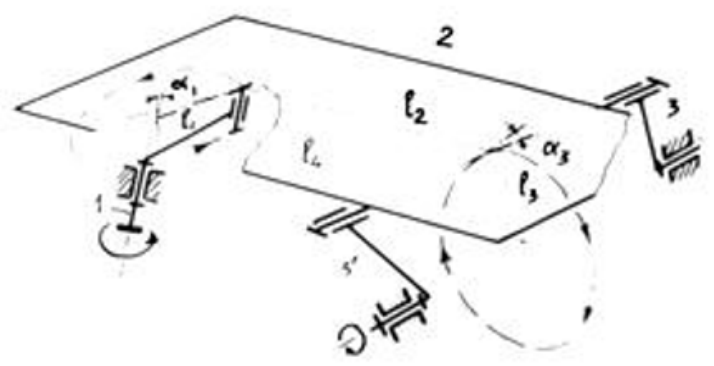

Fig. 2. Kinematic scheme of washing platform drive

3. The use of a connecting rod of mechanisms as a carrier of working elements: a drum in mixers, a cabin in simulators, blades in apparatus with a mixer, etc. Figure 3 shows a kinematic scheme and a photo of a laboratory sample of a mixer of bulk materials, the mixing tank (drum) 2 of which is made on the connecting rod of the basic spatial four-bar mechanism.
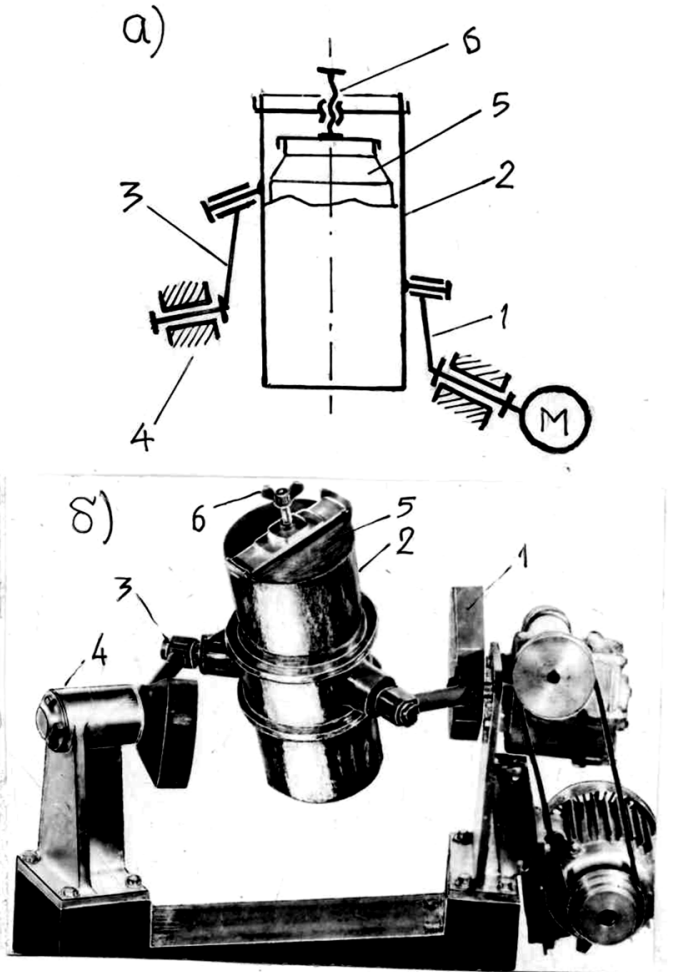

Fig. 3. Screw mixer: a) kinematic scheme b) laboratory sample of a mixer
At the same time, energy consumption is reduced in comparison with existing mixers of the same performance, the time for preparing a homogeneous mixture is reduced by 3-4 times and the final product is ecologicaly pure.

4. The use of machine bars to follow certain paths, such as bird wings, digging bucket, double-grip manipulator, etc. Thus, the use of a connecting rod of a spatial four-bar mechanism with rotational pairs allowed obtaining a mechanical shovel mechanism, where the $\mathrm{M}$ bucket performed the movement of a conventional shovel (Figure 4).

a)

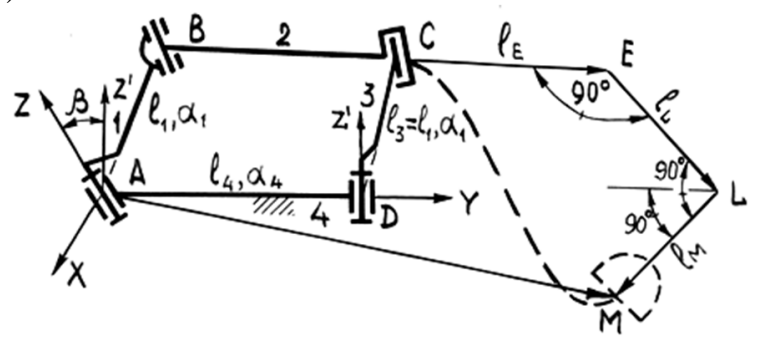

b)

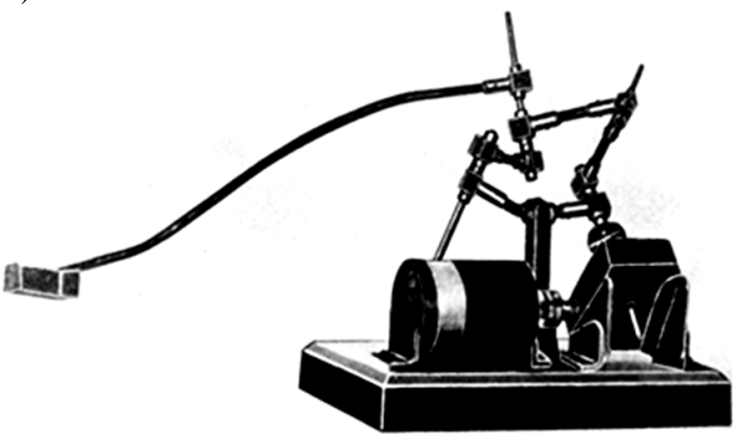

Fig. 4. Mechanical shovel mechanism: a) kinematic scheme b) model

5. The use of bars of mechanisms to intensify mixing processes in mixers, where the bars perform the function of working bodies, each moving according to its own kinematic law

6. The creation of directed force impulses used, for example, in devices for driving and pulling piles, inertial motors, devices for compaction of soil and silo, etc.

7. The reproduction of planetary, reciprocating, rotational, elliptical and other movements.

8. The use of Bennett's mechanism for the formation of spatial multi-bar mechanisms: fivebar, six-bar, seven-bar and differential mechanisms.

Figure 5 shows a spatial six-bar mechanism obtained by sequentially combining three Bennett mechanisms; each of them has two equal bars in the parameters.

The degree of mobility of the six-bar is equal to one and the parameters are found by the ratio:

$$
\frac{l_{1}}{\sin \alpha_{1}}=\frac{l_{2}}{\sin \alpha_{2}}=\frac{l_{3}}{\sin \alpha_{3}}=\frac{l_{4}}{\sin \alpha_{4}}
$$


where $l_{1}=l_{5}, \alpha_{1}=\alpha_{5}$.

a)

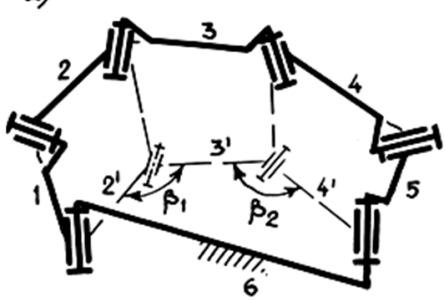

反)

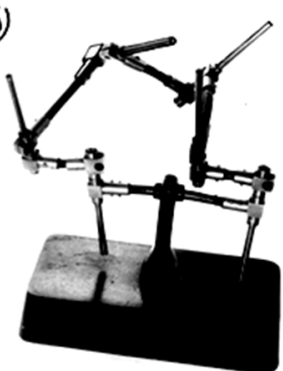

Fig. 5. Six-link spatial mechanism with rotational pairs: a) kinematic scheme b) model

\section{Conclusion}

1. Over recent years, the staff of the Department of the Department of Theory of Mechanisms and Machines (now the Department of General Engineering Disciplines) of Kazan State Agrarian University have created a new direction in the theory of spatial mechanisms, which is newand priority in the world and our country.

2. The new direction was fully developed: it was formed and researched not only theoretically, but also the production samples in various fields of technology and technologies were produced and introduced.

3. The relevance and effectiveness of the use of spatial mechanisms with rotational pairs in technology is confirmed by the fact that 4 doctoral and 10 candidate dissertations were defended on this topic by the staff of the department, 9 monographs were published, 123 inventions were developed, 1 silver and 3 bronze medals of the USSR Exhibition of Economic Achievements were awarded.

4. The devices based on the mechanisms havehigh intensity of processes, low energy consumption, environmental friendliness and reliability. Since the Department of Theory of Mechanisms and Machines (now the Department of General Engineering Disciplines) of Kazan State Agrarian University has the world priority of creation, research and implementation of mechanisms of this group, an international scientific and practical conference « 100 years of Bennett's mechanism » was held in Kazan State Agricultural Academy (KSAU) in 2003. This conference summed up the development of spatial mechanisms for the indicated period $[10,11]$.

\section{References}

1. V.P. Goryachkin, Collected Works. vol. II. Agricultural mechanics (Selkhozgiz, Moscow, 1937) $258 \mathrm{p}$.

2. N.I. Klenin, S.N. Kiselev, A.G. Levshin, Agricultural machines. Agricultural equipment (Kolos-s, Moscow, 2018) 816 p.

3. A.P. Tarasenko, Rotary Combine Harvesters (Lan, St. Petersburg, 2018) 192 p.

4. E.V. Truflyak, E.I. Trubilin, Modern combine harvesters (Lan, St. Petersburg, 2020) 320 p.

5. G.D. Ananov, Kinematics of spatial hinge mechanisms of agricultural machines (Mashgiz, Leningrad, 1963) 220 p.

6. N.I. Klenin, Agricultural and reclamation machines: a textbook for universities (Kolos, Moscow, 2016) 293 p.

7. E.I. Vorobiev, F.M. Diemenberg, Spatial hinge mechanisms. Closed and open kinematic chains (Nauka, Moscow, 1991) 264 p.

8. P.G. Mudrov, Spatial mechanisms with rotating pairs (Kazan University Publishing House, Kazan, 1976) $264 \mathrm{p}$.

9. A.G. Mudrov, Spatial mechanisms with a special structure (RIC "School", Kazan, 2003) 300 p.

10. 100 years of Bennett's mechanism: Mat. of the int. conf. on the theory of mechanisms and machines (RIC "School", Kazan, 2004) 292 p.

11. A.P. Mudrov, A.G. Mudrov, S.M. Yakhin, N.Z. Mingaleev, G.V. Pikmullin, Study of spatial hinge mechanisms and their use in agricultural machines, BIO Web of Conferences, 17, 00012 (2020) 